\title{
Erinnerungspolitik und Medialisierung von DDR-Geschichte. Plädoyer für eine andere Wahrnehmung ${ }^{1}$
}

\author{
Carola Hähnel-Mesnard
}

Université de Lille

\section{Abstract}

Der Beitrag hinterfragt die Konstruktion und Vermittlung der Erinnerung an die DDR. Gegenwärtig ist die Interpretation der DDR-Geschichte in den Medien, in den meisten staatlichen Museen sowie in erfolgreichen Kinoproduktionen immer noch stark durch das totalitaristische Paradigma geprägt. Diese Darstellung steht im Widerspruch zur Alltagswahrnehmung der meisten ehemaligen DDR-Bürger. Obwohl sich die Forschung seit langem für Aspekte der Alltagsgeschichte interessiert, sind solche Elemente in den meisten aktuellen Darstellungen der DDR kaum vorhanden. Andreas Dresens und Laila Stielers Film Gundermann (2018) ist ein Beispiel für den Versuch von Ostdeutschen, die Deutungshoheit über ihre Geschichte zurückzugewinnen. Insgesamt beeinflusst die Unterrepräsentation der Ostdeutschen in Schlüsselpositionen der Gesellschaft auch die Möglichkeiten, auf die öffentlichen Erinnerungsprozesse Einfluss zu nehmen.

Cette contribution s'interroge sur la façon dont la mémoire de la RDA est construite et transmise. Aujourd'hui, l'interprétation de l'histoire de la RDA se fait toujours essentiellement à partir du paradigme totalitaire, que ce soit dans les médias, dans la plupart des musées financés par l'État ou dans les productions cinématographiques à succès. Cette représentation est en décalage par rapport à la perception de la RDA telle qu'elle fut vécue de l'intérieur par la grande majorité de ses citoyens. Alors que la réflexion sur l'histoire du quotidien est présente depuis longtemps dans le monde de la recherche, les aspects qui en relèvent sont peu présents dans les représentations actuelles de la RDA. Le film Gundermann (2018) d'Andreas Dresen et Laila Stieler est un récent exemple d'une tentative entreprise par les Allemands de l'Est pour récupérer un pouvoir d'interprétation de leur passé. Globalement, la sous-représentation de ces derniers dans les positions-clés de la société influe également sur les possibilités de prise de parole sur ce passé.

This paper examines the construction and mediation of the GDR memory. Today, the interpretation of the GDR history in the media, through most of the state museums as well as successful movies, is still strongly influenced by the totalitarian paradigm. This representation contradicts the everyday perception of most former GDR citizens. Although research inspired by Alltagsgeschichte has long been interested in aspects of everyday history, such elements are scarcely present in most current representations of the GDR. Andreas Dresen's and Laila Stieler's film Gundermann (2018) is an example of East German attempts to regain sovereignty over the interpretation of their history. Overall, the under-representation of the East Germans in key positions in society also influences the possibilities of impacting the portrayal of this past.

\section{Keywords}

Darstellung der DDR • Totalitarismus • Alltagsgeschichte $•$ Gundermann • Deutungshoheit

Représentation de la RDA • totalitarisme • histoire du quotidien • Gundermann • pouvoir d'interprétation

Representation of GDR • totalitarism • Alltagsgeschichte $•$ Gundermann • interpretative authority

Spaziert man 2019 im Stadtzentrum von Sofia den schönen Witoscha-Boulevard entlang, stößt man auf einen großen Park, an dessen Ende ein riesiges Gebäude aus der sozialistischen Ära thront: Es ist der ehemalige Kulturpalast, der Ende der 1970er Jahre erbaut wurde und heute als Kongresszentrum und Konzertsaal genutzt wird. Als Bulgarien 2018

1 Übersetzung von Marie-Therese Mäder des Artikels von Carola Hähnel-Mesnard (2020): "La RDA dans le (rétro)viseur. Plaidoyer pour une autre perception“, in Symposium Culture@Kultur (2), 1-7. den Vorsitz im Rat der Europäischen Union innehatte, fanden die offiziellen Treffen in diesem Gebäude statt. Spaziert man 2019 im Stadtzentrum von Berlin den nicht weniger schönen Boulevard Unter den Linden entlang, stößt man auf ein riesiges Gebäude aus einem unbestimmten Zeitalter, das sich als Schloss aus dem 18. Jahrhundert ausgibt, ehemalige Residenz der Hohenzollern und Symbol des ,Besten', was die absolute Monarchie Preußens zu bieten hatte. Doch was geht in einem Besucher vor, der seit Jahren nicht in Berlin war und sich noch an ein anderes riesiges Gebäude aus der sozialistischen Ära erinnert, das Ende der 1970er Jahre am 
selben Ort errichtet wurde? Er wird ungläubig seine damals aufgenommenen Fotos mit dem neuen Bauwerk abgleichen und vergeblich nach einem auch noch so kleinen Hinweisschild suchen, das inm anzeigt, dass hier zwischen 1976 und 2006 ganz offiziell der Palast der Republik stand, und er wird vielleicht zu dem Schluss kommen, dass er einer Sinnestäuschung unterlag oder die Kameras der damaligen Zeit doch sehr unzuverlässig waren.

Sofia und Berlin: Der vergleichende Blick führt plötzlich noch einmal vor Augen, wie Teile der ostdeutschen Lebenswelt und der DDR-Geschichte einfach verschwunden sind, so als hätte diese Geschichte nie stattgefunden. Dieses „Verwischen der Spuren" wurde in verschiedenen Untersuchungen beschrieben, auch in Frankreich, und muss hier nicht weiter thematisiert werden (u.a. Robin 2001; Combe / Dufrêne / Robin 2009; Goudin 2017). Den Sofiotern jedenfalls wurde die Zuständigkeit für diesen Teil ihrer Geschichte nicht abgesprochen und sie werden auch in Zukunft ganz allein über das Schicksal der sozialistischen Ära in ihrem kollektiven Gedächtnis entscheiden.

Dass den Ostdeutschen die Zuständigkeit für ihre Geschichte abgesprochen wurde - mancher wird denken, dass dies große Worte sind. Dennoch sollte man sich für den Umgang mit der DDR-Geschichte genauer interessieren, wenn man sich fragt, warum sich dreißig Jahre nach dem Mauerfall die innere Einheit in Deutschland noch immer nicht eingestellt hat und was die Gründe für das Unbehagen in den ,neuen' Ländern sind, welches sich in einem massiven Votum für die rechtsextreme und populistische Partei AfD ausdrückt. Im Folgenden soll deshalb interessieren, wie DDR-Geschichte in der deutschen Öffentlichkeit debattiert und erinnert wird und wie ehemalige DDR-Bürger daran teilhaben können. Letztlich geht es dabei um nicht mehr und nicht weniger als um die Frage, wie sich der Osten in die gesamtdeutsche Gesellschaft einzubringen vermag

\section{VOM GESPENST DES TOTALITARISMUS ZUM GEFÜHL DER UNZULÄNGLICHKEIT DER ERINNERUNGSPOLITIK}

Als im September 2018 der Comic Spirou in Berlin von Flix herauskam, widmete ihm das ARTE Journal einen kurzen Bericht (Frank 2018). So erfährt man, dass Flix, bevor er seine Helden Spirou und Fantasio auf einige Abenteuer in das Ostberlin der 1980er Jahre schickt, sich die Mühe gemacht hatte, einige Informationen einzuholen. Um das im Comic wiederkehrende Motiv der dressierten Affen verwenden zu können, entdeckt der Zeichner, dass dressierte Affen auch in der DDR eine Rolle spielten! Tatsächlich gab es im Berliner Tierpark eine Affenzucht. Bis hierhin nichts Ungewöhnliches für einen auch noch so kommunistischen Zoo. Natürlich geht die Überlegung weit darüber hinaus: "Gleichzeitig ist der dressierte Affe ja auch so ein Symbol für Menschen in einem geschlossenen politischen System, wie es die DDR eins war." Nun gut. Im Anschluss daran teilt uns Flix mit, er habe für den Band versucht, „genau zu recherchieren, welche Art von Bedrohung [...] es für die Bürger in der DDR [gab]. Eine Möglichkeit, was ihnen passieren [konnte], wenn sie sich nicht konform verhalten haben, war, dass sie verhaftet wurden." Im Gefängnis waren sie dann „einer psychischen und physischen Folter ausgesetzt." Währenddessen wird Flix im Stasi-Gefängnis in Hohenschönhausen gefilmt, die französische Stimme aus dem Off betont das Wort „Folter“, „torture“. Man hätte sich ein wenig mehr Informationen über die genaueren Umstände gewünscht. Und was es bedeutet, sich „nicht konform zu verhalten“, um ins Gefängnis zu kommen. Der Ton ist vorgegeben, ebenso wie die Handlung einer Geschichte, die sich seit dreißig Jahren unablässig wiederholt: Die Ostdeutschen, deren Rückgrat so nachgiebig ist wie das eines Affen, sind Opfer eines totalitären Systems, das sie bei der geringsten Gelegenheit einsperrt. Für Flix, 1976 in Münster in der Bundesrepublik geboren, endet dort seine kurze Auseinandersetzung mit der Geschichte der DDR.

Warum muss man nun ausgerechnet diesem kaum zwei Minuten langen Bericht, der im deutsch-französischen Kulturkanal ausgestrahlt wurde, eine solche Bedeutung zumessen? Weil er stellvertretend für einen herablassenden Diskurs sowie für eine voreingenommene und einseitige Sicht auf die ostdeutsche Gesellschaft und Geschichte steht und weil er einen immer häufiger anzutreffenden historischen Relativismus vertritt. Wenn man bei jeder Gelegenheit unpräzise auf das totalitäre Paradigma zurückgreift, dann werden dadurch die Unterschiede zwischen dem Terror eines totalitären Regimes wie im Falle des Nationalsozialismus oder Stalinismus und dem Kontroll- und Überwachungsapparat einer autoritären Diktatur nivelliert.

In ihrem neuesten Buch, einer virulenten und anregenden Abrechnung mit dem vereinten Deutschland, prangert die ostdeutsche Essayistin Daniela Dahn die undifferenzierte und immer wiederkehrende Verwendung des Begriffs „totalitär" hinsichtlich der DDR an und erinnert dabei an die Worte Hannah Arendts, die in ihrem Vorwort zur dritten Ausgabe der Elemente und Ursprünge totaler Herrschaft von 1966 davor warnte, dass der damals weit verbreitete Antikommunismus es verbiete, „die verschiedenen kommunistischen Einparteiendiktaturen [...] von einem echten totalitären Regime zu unterscheiden“, obwohl man allen Grund hätte, „mit dem Wort ,totalitär" sparsam und vorsichtig umzugehen“ (zitiert nach Dahn, 2019, 100f.). Die Historikerin Sonia Combe beklagt ihrerseits eine Memorialisierung der DDR-Vergangenheit, die zunehmend dazu neige, den Naziterror mit der ostdeutschen Diktatur gleichzusetzen und Kontinuitäten von 1933 bis 1989 nahezulegen, wie im Fall der oben genannten Gedenkstätte des Stasi-Gefängnisses in Hohenschönhausen (Combe 2019).

Die öffentliche Erinnerung an die DDR und ihre Darstellung in Museen und Gedenkstätten ist seit langem Gegenstand von Debatten. Doch haben die immer gleichen Diskussionen bislang zu keinem wesentlichen Perspektivwechsel geführt. Denn bei den meisten Museen und Gedenkstätten handelt es sich um die ehemaligen Stätten der Macht, der Überwachung und Repression: Stasi-Museen, Gefängnisse, Berliner Mauer und Grenzposten. Diese Beobachtung machten 2006 der 
Historiker Martin Sabrow und die von inm geleitete Expertenkommission, die seinerzeit für die Erarbeitung eines globalen Entwurfs zur „Schaffung eines Geschichtsverbundes ,Aufarbeitung der SED-Diktatur"“ berufen wurde (Sabrow et al. 2007: 17-43). Der Kommission zufolge sei es notwendig, über einen solchen machtzentrierten Ansatz hinauszugehen, dem Alltagsleben mehr Raum zu geben und die individuellen Verhaltensweisen der Akteure der Gesellschaft in ihren Beziehungen zur Macht zu hinterfragen, Verhaltensweisen, die zwischen „Akzeptanz und Auflehnung, Begeisterung und Verachtung, mißmutiger Loyalität und Nischenglück" oszillieren konnten (ebd.: 31f.). In dieser Hinsicht machte sich die Sabrow-Kommission zum Sprachrohr eines wichtigen Teils der universitären Forschung, die sich seit den 1990er Jahren von der stark von Totalitarismustheorien beeinflussten und sich ausschließlich für Institutionen und Machtstrukturen interessierenden Politikgeschichte abwendete. Es ging nunmehr darum, einer Sozial- und Alltagsgeschichte den Vorrang zu geben, die sich von der Vorstellung einer monolithischen Gesellschaft distanziert und eine Diskrepanz zwischen Herrschaftswillen und gelebter Erfahrung voraussetzt (Lindenberger 1999; Kott / Droit 2006). Die Sabrow-Kommission schlug daher vor, die „Bindungskräfte“ zu untersuchen, die es dem Regime in den 1960er und 1970er Jahren erlaubten, eine gewisse Stabilität zu erlangen. Diese „Bindungskräfte“ reichten von ideologischer Überzeugung bis hin zu Möglichkeiten des sozialen Aufstiegs und der wirtschaftlichen Sicherheit (Sabrow et al. 2007: 34). Diese Ansätze stellten in keiner Weise das Gedächtnis der Opfer der Repression des Regimes in Frage, sondern fügten lediglich einen Pfeiler hinzu, um die dem politischen System innewohnenden Widersprüche zum Ausdruck zu bringen. So sollte den Bürgern, die meinten, in der DDR ein „normales Leben“ geführt zu haben (Fulbrook 2008) - wohl die große Mehrheit der Bevölkerung - Raum zur Reflexion geboten und gleichzeitig eine nachträgliche Idealisierung der Vergangenheit verhindert werden.

Die Empfehlungen der Kommission wurden nicht befolgt und man warf ihr vor, die vom ostdeutschen Regime begangenen Taten zu verharmlosen. Die Stasi würde die DDR besser charakterisieren als die Kinderkrippen, so lautete das unvergessene Urteil von Horst Müller, zum damaligen Zeitpunkt Direktor des Instituts für Zeitgeschichte in München, (ebd.: 56). Seitdem ist die Debatte nicht vorangekommen. Als sich der Politologe Klaus Christoph im Jahr 2013 noch einmal rückblickend dem Thema der Aufarbeitung der DDR-Diktatur widmet, stellt er fest, dass sich die Regierungsberichte von einer Parlamentsdebatte zur nächsten ähneln und die Erinnerungspolitik weiterhin den unmenschlichen Charakter der kommunistischen Diktatur und den Dualismus zwischen Opfern und Tätern hervorhebt. Ganz im Sinne der Sabrow-Kommission weist der Politologe darauf hin, dass sich die Mehrheit der ostdeutschen Bevölkerung in diesem Muster nicht wiedererkennt und dass die individuellen Erfahrungen meist im Widerspruch zu dem von den politischen Instanzen vermittelten Geschichtsbild stehen. Und er verweist auf eine der möglichen Folgen dieses Mangels an Zwischentönen: die „Wiederbelebung einer problematischen Grunderfahrung des ,gelernten“ DDR-Bürgers“, die darin bestand, mit einer vom „SED-Apparat inszenierten Wirklichkeitsdeutung" konfrontiert zu sein, die mit der Realität und der eigenen Erfahrung nur wenig zu tun hatte (Christoph 2013).

Im Jahr 2019 drückt sich dieses Gefühl der Diskrepanz noch auf eine andere Art und Weise aus, nämlich in einer starken Zustimmung zu einer anti-elitären und anti-demokratischen Rhetorik, in der das heutige Deutschland nicht selten mit der DDR verglichen wird. In dem kürzlich erschienenen Buch Wer wir sind, in dem der Soziologe Wolfgang Engler und die Journalistin Jana Hensel versuchen, die ostdeutschen Erfahrungen der letzten drei Jahrzehnte zu erklären, bringt Hensel die Marginalisierung der Ostdeutschen bei der Interpretation ihrer Geschichte zur Sprache. Man habe das Gefühl, „die Erzählung der eigenen Geschichte haben andere übernommen“ (Engler / Hensel 2018: 192). Gegenwärtig lässt sich insofern ein kleiner Perspektivwechsel beobachten, als der Zuwachs rechtsextremer Wählerstimmen in den neuen Bundesländern dazu veranlasst, die Gründe dieses Wahlverhaltens zu hinterfragen. Und da uns nun dreißig Jahre von einer DDR trennen, die nur vierzig Jahre existiert hat, beginnt man einzuräumen, dass die Gründe für dieses Votum nicht mehr in der Geschichte der DDR zu suchen sind, sondern in den Erfahrungen seit 1990 (ebd.: 51). Es bleibt abzuwarten, ob die Politik auch Irrtümer in der offiziellen und öffentlichen Erinnerungskultur eingestehen und daraus Konsequenzen ziehen wird.

\section{HELDENGESCHICHTEN VERSUS ERINNE- RUNG AN DEN ALLTAG}

Ein wichtiger Träger des öffentlichen Erinnerns an die DDR sind Fernsehen und Kino (Camarade / Guilhamon u.a. 2018). Wie anhand des ARTE-Beispiels bereits ausgeführt wurde, ist auch hier die Darstellung der DDR selten differenziert und kann beim ostdeutschen Publikum ein Gefühl der Fremdheit hervorrufen. In einem Interview aus dem Jahr 2016 argumentiert der ostdeutsche Filmemacher Thomas Heise, dass die Stasi das Bild der DDR zu lange dominiert habe, und dass die DDR immer noch als "monolithischer Block ohne eigene Geschichte“ wahrgenommen werde, während die Realität im Jahr 1949 oder 1989 nicht die gleiche war (Steinle 2018: 304).

Zu den Filmen, die auch im Ausland großen kommerziellen Erfolg haben, gehören Spielfilme über die Stasi, wie Das Leben der Anderen (2006), aber auch Heldengeschichten über den Widerstand gegen das Regime oder über Fluchtversuche aus der DDR. So sind kürzlich Das schweigende Klassenzimmer von Lars Kraume (2018) und Ballon von Michael Herbig (2018) auch in Frankreich in die Kinos gekommen. Der erste Film erzählt von den Folgen einer Schweigeminute, die eine Abiturklasse abhält, um so gegen die sowjetische Niederschlagung des Aufstands von 1956 in Ungarn zu protestieren. Obwohl das Verhältnis zwischen Bürgern und Staat durchaus differenziert dargestellt wird, spielt der Film von Anfang an bewusst und unter Rückgriff auf zahlreiche Klischees 
mit Analogien zwischen dem ostdeutschen Machtapparat und den Nationalsozialisten - es kommt auch hier zu einer problematischen Gleichstellung beider Regime. Der zweite Film erzählt die Geschichte der Flucht zweier ostdeutscher Familien in einem Heißluftballon, eine Story, die bereits Anfang der 1980er Jahre von Disney verfilmt wurde. Wie sein Kollege Lars Kraume wurde der Schauspieler und Komiker Michael Herbig in der Bundesrepublik geboren. Ihre jeweiligen Filme basieren auf ,wahren Geschichten', was innen eine gewisse Authentizität verleiht. Doch diese Filme sind weit davon entfernt, den Alltag eines DDR-Bürgers abbilden zu können, sie beruhen auf Sensation und Außergewöhnlichem, und es wird dem durchschnittlichen Ostdeutschen schwerfallen, sich darin wiederzufinden. Die große Mehrheit vermied es, den Machtinstanzen zu nahe zu kommen oder ihr Leben bei Fluchtversuchen aufs Spiel zu setzen. Natürlich ist es Kino und zum Kino gehören außergewöhnliche Geschichten, die zum Träumen anregen oder Schauder provozieren. Doch wird diese Art von Film dank einer realistischen und Retro-Ästhetik meist als historisches Epochendokument wahrgenommen, als adäquate und erschöpfende Darstellung einer vergangenen Zeit. Die Protagonisten, deren angenehmes Äußeres der sorgfältig rekonstruierten Kulisse in nichts nachsteht, erlauben Empathie und ermöglichen es dem Zuschauer, sich mit ihnen zu identifizieren (Mesnard 2018: 10). In Deutschland und Frankreich werden diese aus kinematografischer und ästhetischer Sicht oft banalen Filme auch zu pädagogischen Zwecken eingesetzt. Begleitet von umfassendem Lehrmaterial vermitteln sie Generationen von Schülern ein bestimmtes Bild der Vergangenheit. Auch wenn sich diese ,Unterrichtsquellen“ in Abhängigkeit von den neuesten Produktionen des Marktes immer wieder ändern, bleiben die Erzählstruktur und der Ton gleich: Die DDR war ein repressiver Staat, aus dem die Bürger zu fliehen versuchten. Bereits in den 1960er Jahren war die westliche Vorstellungswelt über die DDR um die Stichworte ,Mauer' und ,Flucht' aufgebaut worden, und die Mehrzahl der jüngsten Filme spiegeln dies noch immer wider. Andere Fragen zu dieser Zeit tauchen gar nicht erst auf.

Es interessiert also nicht, wie die Menschen im Alltag gelebt haben, welche Sorgen sie in der geschlossenen Gesellschaft hatten, wie ihr Verhältniss zur Macht außerhalb einer direkten Konfrontation aussah, wie sie sich mit der Situation begrenzter Freiheit und relativen Zwangs einrichteten und wo sie ihre eigenen Spielräume, ihre Freiheiten finden konnten. Gleiches gilt für die Erinnerungskultur. Die DDR war keine Kombination aus Schwarz-Weiß und allerlei Abstufungen von Grau, wie es allgemein dargestellt wird. Was dem Kassenschlager-Kino fehlt, das den Anspruch erhebt, die Vergangenheit der DDR nachzuzeichnen, ist die Darstellung eines Gedächtnisses des Alltags und der sozialen Beziehungen. Das ostdeutsche Kino selbst ist dafür eine großartige Quelle. Ob es sich dabei um Spiel- oder Dokumentarfilme handelt, die DEFA-Produktionen sind „eine unerlässliche Informationsquelle für alle, die nicht nur etwas über heutige Erinnerungen an die DDR erfahren wollen, sondern auch über eine ,von innen gelebte' DDR nachdenken wollen“, wie Caroline Moine vor einiger Zeit bemerkt hatte (Moine 2006: 171).

Filme, die die DDR differenziert ,von innen' zeigen, sind ebenso selten wie solche, die sich mit den sozialen Folgen der Wiedervereinigung beschäftigen. Und sie sind weit weniger sichtbar. Während Florian Henckel von Donnersmarcks erster Spielfilm Das Leben der Anderen im Jahr 2006 Millionen von Zuschauern anzog, war Bernd Böhlichs 2007 erschienene Gesellschaftskomödie Du bist nicht allein zwar ein kleiner Erfolg in Deutschland, wurde aber anderswo nicht rezipiert. Natürlich sind die neuen Arbeitslosen in den Plattenbauten Ostberlins weniger faszinierend als der ambivalente Stasi-Offizier. Dies trifft auch auf zwei wichtige Filme zu, die 2018, d.h. zur gleichen Zeit wie die oben erwähnten ,Heldengeschichten', angelaufen sind. Es ist im Übrigen aufschlussreich, das gewählte Thema mit der Herkunft der Regisseure zu vergleichen: Der Osten dreht Filme sozialer Natur, der Westen die anderen. Thomas Stubers Spielfilm In den Gängen, basierend auf einer Kurzgeschichte von Clemens Meyer, ist fast ausschließlich hinter den verschlossenen Türen eines Großmarktes in der ostdeutschen Provinz gedreht, wo der Protagonist Christian den Beruf des Gabelstaplerfahrers erlernt. Die Größe des Supermarktes und die langen, mit Waren gefüllten Gänge sind das genaue Gegenteil der Enge eines Alltags und einer beruflichen Tätigkeit, die jede Bedeutung verloren haben, wenn man von den menschlichen Beziehungen einmal absieht, die dort aufgebaut werden. So lernt Christian Bruno kennen, einen viel älteren Mitarbeiter, der inn unter seine Fittiche nimmt. Es ist diese scheinbar äußerst stabile Figur, die die ganze Gewalt der nach 1989 eingetretenen Veränderungen in sich zusammenfasst. Einst Lkw-Fahrer in der DDR, hat er seinen Job verloren und lässt Christian wissen, wie sehr inm die Straße fehlt. Mehr wird nicht gesagt, aber sein Freiheitsgefühl auf der Straße steht der neuen Konsumfreiheit diskret gegenüber. Bruno, Abteilungsleiter, anscheinend gut integriert, wird Selbstmord begehen, niemand hat damit gerechnet. Abgesehen von der Tatsache, dass der Film ein Bild der Unterschichten in der heutigen ostdeutschen Provinz zeichnet, suggeriert er nichts anderes, als dass das Leben in der DDR Sinn ergeben konnte und dass die Implosion der alten Lebenswelt Spuren hinterlässt, auch noch lange nach ihrem Verschwinden.

Das eigentliche Kinoereignis des Jahres war Gundermann (2018), ein Film von Regisseur Andreas Dresen und Drehbuchautorin Laila Stieler. In Frankreich wird der Film nicht vertrieben und er wurde nur bei seltenen Gelegenheiten gezeigt, so anlässlich der Gedenkfeiern zum 30. Jahrestag des Mauerfalls. Ein Film also, den kein französischer Lehrer in seinem Geschichts- oder Deutschunterricht verwenden wird. Natürlich ist die Geschichte komplex und beschränkt sich nicht auf die Schwarz-Weiß-Muster, an die das Publikum gewöhnt ist. Der biografische Film zeichnet das Leben des ostdeutschen Liedermachers Gerhard Gundermann (1955-1998) nach, der im „wirklichen Leben“ Fahrer eines riesigen Baggers in einem Braunkohletagebau war. Der Film konzentriert sich auf 
die Zeit der Transformation in den 1990er Jahren, bietet aber gleichzeitig durch Flashbacks einen Einblick in die ostdeutsche Gesellschaft vor dem Fall der Mauer, wobei die Gesellschaft jedes Mal von innen heraus beschrieben wird.

Gundermann wird als ebenso vielseitige wie ambivalente Persönlichkeit porträtiert: ein einfacher Arbeiter im Schichtdienst, die übrige Zeit Künstler. Den Parteifunktionären gegenüber verhält er sich widerspenstig und hält dennoch an der Sache des Sozialismus fest. Aus der Partei wird er ausgeschlossen und von der Stasi, der er seine Beschwerdehefte über die Arbeitsbedingungen im Tagebau vorlegt, rekrutiert, bis diese schließlich auf seine Dienste verzichtet und inn selbst überwacht. Als Gundermanns Zusammenarbeit mit der Stasi in den 1990er Jahren öffentlich wird, weigert er sich, das übliche Opfer-Täter-Schema zu bedienen. Genau darin besteht die Absicht des Films: auf vereinfachende Leseraster zu verzichten, endgültige Urteile abzulehnen und den ostdeutschen Biografien ihre Komplexität wiederzugeben. Gundermann ist ein wichtiger Film, weil eine ostdeutsche Drehbuchautorin und ein ostdeutscher Regisseur, geboren in den 1960er Jahren, die Geschichte der DDR auf ihre eigene Weise erzählen. Dresen erklärt im Begleitheft der DVD, dass beide es leid waren, ständig mit Klischees und Stereotypen konfrontiert zu werden und endlich die Deutungshoheit über ihre eigene Geschichte zurückgewinnen wollten. Ein Film im Übrigen, für den es sehr schwierig war, eine Finanzierung und einen Produzenten zu finden. Nicht nur, dass der Liedermacher Gundermann dem westdeutschen Publikum völlig unbekannt war, auch die besondere Dramaturgie und das Drehbuch verstießen gegen die Konventionen des Genres, indem sie die „Läuterung“ der Figur verweigerten (Dresen/ Stieler 2018). Es versteht sich von selbst, dass Ballon nicht die gleichen Probleme hatte.

Gundermann ist nicht nur ein Film darüber, wie sich der Einzelne gegenüber der sozialistischen Idee positioniert und wie die Vertreter der Macht sie pervertieren, es ist auch ein Film, der minutiös die Arbeitswelt in der DDR nachzeichnet, die Brigadearbeit, die Schwierigkeiten, mit teilweise veralteter Ausrüstung die Vorgaben des Wirtschaftsplans zu erfüllen, die Genugtuung, dass es trotz minderwertiger Rohstoffe gelingt, das Land mit Heizmaterial zu versorgen. Besonders bewegend ist die Figur der Helga, eine kleine zarte Frau, die majestätisch den riesigen Bagger führt und auf die Rolle der Frauen in diesem Arbeitermilieu verweist. Was Gundermann betrifft, so hat er das Bedürfnis, vor und nach seinen Konzerten in seinem Tagebau arbeiten zu gehen. Selbst als er in den frühen 1990er Jahren immer berühmter wird und von seiner Musik hätte leben können, will er sich seine Freiheit gegenüber der Unterhaltungsindustrie bewahren. Er und seine Kollegen sind von der Schließung des Tagebaus und der daraus resultierenden Arbeitslosigkeit besonders betroffen. Damit reicht der Film von der Darstellung der Arbeitswelt in der DDR bis hin zu deren Auflösung in den 1990er Jahren, denn die Industrie in den neuen Bundesländern überlebte die Ankunft der neuen Marktwirtschaft nur in den seltensten Fällen. In einem der Lieder, die dem Film seinen ganz besonderen Rhythmus verleihen, drückt Gundermann das Gefühl der Herabstufung und Verzweiflung aus, das die Zeit nach 1989 kennzeichnet und sich heutzutage in Ressentiments verwandelt hat: „Hier bin ich geborn / wo die Kühe mager sind wie das Glück /[...]/ hier sind die Nullen ganz unter sich / hier isses heute nicht besser als gestern/und ein Morgen gibt es hier nicht [...]." Das Originallied Hier bin ich geboren stammt aus dem Jahr 1995. Vielleicht hätte sich das Kino früher für Anti-Helden wie Gundermann interessieren sollen, der mit seiner riesigen Brille und seinem aus der Zeit gefallenen Pferdeschwanz nicht zu einer leichten Identifikation einlädt, der aber besser als die anderen Helden die Lebenswelt einer ganzen Epoche verkörpert, mit ihren Zwängen, ihren Handlungsspielräumen und den individuellen Entscheidungen, die man treffen konnte und musste. Ein Anti-Held, der gleichzeitig auch das Verschwinden dieser Lebenswelt verkörpert, ebenso wie die daraus resultierende Trostlosigkeit und die bald eintretende Stummheit.

\section{DIE EIGENE STIMME WIEDERFINDEN}

Im Vorspann von Thomas Heises Dokumentarfilm Material aus dem Jahr 2009, der aus bis dahin unbenutztem Filmmaterial geschnitten wurde, bekräftigt der Filmemacher: „Immer bleibt etwas übrig, ein Rest, der nicht aufgeht, dann liegen die Bilder herum und warten auf Geschichte." Es gibt immer Bilder, die sich der großen Erzählung der Geschichte entziehen und darauf warten, wiederentdeckt zu werden, um ihre eigene Geschichte zu erzählen. Vielleicht müsste man anfangen, die Geschichte auf eine etwas andere Art und Weise zu erzählen? Heises Satz erinnert an Walter Benjamins Lumpensammler, der sich für den Abfall der Geschichte interessiert und damit für jenes, das von ihr ausgeschlossen wurde - und der sich, als Historiker, für die „Unterdrückten“ der Geschichte interessiert. Die Metapher sollte nicht überstrapaziert werden: die Ostdeutschen sind keine Opfer der Geschichte. Bei den ersten freien Wahlen im März 1990 stimmt die Mehrheit von ihnen, beflügelt von den Versprechungen einer raschen Transformation, der Einführung der D-Mark und dem Zugang zum Konsum, für das Bündnis der konservativen Parteien und ebnet damit den Weg für einen extrem schnellen Einigungsprozess, für dessen Folgen bis heute bezahlt wird. Die Mehrheit stimmt also nicht für die Vertreter der Bürgerbewegungen, welche in der Vereinigung der beiden deutschen Staaten keine zutreffende Option sahen und die den Sozialismus reformieren wollten, um einen dritten Weg zu finden. Doch konnte oder wollte sich damals die Mehrheit sicherlich nicht vorstellen, dass das Gegenstück zur D-Mark in den tiefgreifenden Veränderungen lag, die ihr Leben auf den Kopf stellen würden. Diese Mehrheit konnte sich auch nicht vorstellen, dass die soziale Marktwirtschaft, deren Bild ihnen als langjährigen Beobachtern der Bundesrepublik vertraut war, unter den Auswirkungen der immer mehr ausufernden Globalisierung schnell inr ,soziales' Attribut verlieren würde. Die Rolle der Treuhand wirft heute insbesondere Fragen nach dem Ausverkauf der ostdeutschen Industrie und den damit verbundenen Folgen 
auf: die Deindustrialisierung dieser Regionen, die steigende Arbeitslosigkeit, Abwanderung.

Die Mehrheit der Ostdeutschen hätte damals aber sehen können, dass sie bei der Entscheidung über ihre Zukunft und bei der Bestimmung ihrer Optionen nicht mehr allein waren. Tatsächlich, so beschreibt es der Soziologe Steffen Mau, kamen bei diesen ersten demokratischen Wahlen im März 1990 auf den „Marktplätzen der DDR [...] die Hauptredner aus Westdeutschland" - wie im Übrigen die meisten AfD-Führungskräfte heute - und die neuen unabhängigen Parteien hatten weder eine Chance gegen die von ihren westdeutschen Genossen finanzierte und unterstützte SPD bzw. CDU, die den Wahlkampf weitgehend dominierten, noch gegen die neue, auf den Trümmern der SED gegründete PDS (Mau 2019: 122). Hätten die Ostdeutschen ohne diese sehr starke und offensichtliche Einmischung anders gestimmt? Wahrscheinlich nicht. Im Rückblick ist es jedoch der Moment, in dem die Vertreter der Bürgerbewegungen, die die Demokratiebewegung und den Reformprozess in Gang gebracht hatten, ihrer Utopien, derer man sich heute kaum noch erinnert, beraubt wurden. Und es ist der Moment, in dem die Ostdeutschen begonnen haben, die Kontrolle über die Veränderungen zu verlieren, von denen sie doch in erster Linie betroffen waren. Um das heutige Wahlverhalten und das zum Teil überaus starke Ressentiment in Ostdeutschland zu erklären, ist das Problem der Abwertung von Lebenserfahrungen und der Austausch der Eliten nicht zu unterschätzen. Daniela Dahn erinnert an eine Reihe beunruhigender Beispiele, wie unfundierte Gutachten sogenannter Spezialisten, die das Ende ostdeutscher Institutionen einläuteten oder die Ablösung von Führungskräften anmahnten (Dahn 2019: 68-83). Der Soziologe Raj Kollmorgen hat eine Bilanz dieser desaströsen Entwicklung gezogen: Er schätzt, dass Ostdeutsche nur $25 \%$ der Eliten auf ihrem eigenen Territorium, also in den neuen Bundesländern, ausmachen. Was die restlichen 75\% betrifft: Sie kommen aus dem Westen. Auf Bundesebene liegt die Repräsentativität von Ostdeutschen bei etwa $3 \%$ in Führungspositionen. Je höher die Position - Bundesrichter, Armeegeneral - desto weniger ostdeutsche Vertreter gibt es (Kollmorgen 2017: 57f.). Ostdeutsche sind folglich in Schlüsselpositionen der Verwaltung, Justiz, Armee, Medien, Unternehmen und Universitäten in der Minderheit. Kollmorgen betont auch das Phänomen der Selbstmarginalisierung: Im Osten geborene junge Deutsche hätten nicht einmal mehr den Ehrgeiz, wichtige Positionen zu besetzen (ebd.: 62).

Diese Situation der Nichtrepräsentanz von Ostdeutschen in den Entscheidungsgremien - Angela Merkel wird wie Joachim Gauck die Ausnahme von der Regel bleiben führt zwangsläufig dazu, dass ostdeutsche Stimmen kaum gehört werden, was sich auch in der Wahrnehmung des Ostens im öffentlichen Raum widerspiegelt. Selbst kulturelle Orte mit einer anfangs starken ostdeutschen Identität stehen heute meist für Trends aus München, Hamburg oder London, man denke an die Debatten um die Nachfolge von Frank Castorf an der Berliner Volksbühne und an das
Leipziger Dokumentarfilmfestival, das seit 2004 keine aus dem Osten stammende Leitung mehr hatte. Wie der Journalist Matthias Dell bemerkt, könnte man natürlich damit aufhören, ständig auf die Herkunft zu schauen, wenn auch die entsprechenden Institutionen in Westdeutschland von Ostdeutschen geleitet würden. Aber das ist nicht der Fall (Dell 2019).

Auf Ostdeutschland lastet eine symbolische Gewalt der Nicht-Anerkennung, die mit Gleichgültigkeit und Ignoranz verbunden ist. Die meisten Westdeutschen sind letztlich vom Schicksal der östlichen Regionen kaum betroffen, so wie der Mauerfall oder die Zeit der Transformation wenig Einfluss auf ihr Leben hatten. Die Geschichte der DDR sowie die ihrer Kultur und Literatur werden heute vermutlich mehr an Universitäten in Frankreich und den Vereinigten Staaten gelehrt als in Deutschland selbst. Und über die friedliche Revolution von 1989 ist letztendlich wenig bekannt. Vielleicht wird die aktuelle politische Krise, die durch den Aufstieg der extremen Rechten in den neuen Bundesländern ausgelöst wurde, zu einem neuen Bewusstsein führen. In jüngster Zeit veröffentlichte Bücher versuchen auf jeden Fall immer wieder, den ostdeutschen Blick auf die letzten dreißig Jahre zu erklären (Engler / Hensel 2018; Dahn 2019; Mau 2019).

Im Jahr 1990 wies der Essayist Lothar Baier darauf hin, dass die Westdeutschen auf dem Gebiet der DDR einen Raum fanden, in dem sie ohne große Mühe einen Teil ihrer „versäumten Kolonialgeschichte" nachholen konnten, ganz ohne anstrengende Eroberungszüge und ohne dafür einen hohen Preis zahlen zu müssen (Baier 1990: 105)². Zugleich warnte er vor dem „Bumerang“-Effekt, den eine „schnelle Kolonisierung" der DDR in der Zukunft verursachen könnte (ebd.: 111). Diesen „Bumerang“-Effekt bekommen wir, wie es scheint, im Moment voll zu spüren. Man könnte den kolonialen oder postkolonialen Vergleich auch aus einer anderen Perspektive bemühen. Man könnte mit Gayatri Spivak fragen: Können die Ostdeutschen „sprechen“? Can the Subaltern speak? An die Stelle des Untergebenen gesetzt zu werden, ist im Osten ein weit verbreitetes Gefühl, paradoxerweise sogar dort, wo der Untergebene Erfolg hat. Kollmorgen erinnert daran, dass Ostdeutsche und Ostdeutschland auch heute noch als dem Westen unterlegen gelten (Kollmorgen 2017: 64). Der Mangel an positiver Sichtbarkeit im öffentlichen Raum und der Eindruck, dass immer andere für einen sprechen, das Gefühl, stigmatisiert und herablassend betrachtet zu werden, all das hat Folgen für das Selbstbild. Im Moment sprechen die „Untergebenen" vor allem über die Wahlurne, es ist an der Zeit, dass sie wieder einen sichtbaren Platz in der Gesellschaft einnehmen, um ihre Stimme auf andere und konstruktive Weise einzusetzen, vorausgesetzt natürlich, dass man sie sprechen lässt.

2 Diese „Kolonisierungsthese“ war in der Literatur unmittelbar nach der Wende sehr präsent, verteidigt von ostdeutschen Autoren wie Volker Braun, Christoph Hein, Stefan Heym, Heiner Müller und Christa Wolf, aber auch von westdeutschen Autoren wie Günter Grass, Rolf Hochhuth und F.C. Delius (Born 2019: 94-116, 218). 


\section{Bibliographie}

Baier, Lothar (1990), Volk ohne Zeit. Essay über das eilige Vaterland, Berlin, Wagenbach.

Camarade, Hélène / Guilhamon, Elizabeth / Steinle, Matthias / Yèche, Hélène (Hrsg.) (2018), La RDA et la société postsocialiste dans le cinéma allemand après 1989, Villeneuve d'Ascq, Septentrion.

Born, Arne (2019), Literaturgeschichte der deutschen Einheit 19892000. Fremdheit zwischen Ost und West, Hannover, Wehrhahn Verlag.

Christoph, Klaus (2013), „Aufarbeitung der SED-Diktatur' heute so wie gestern? - Essay", in Aus Politik und Zeitgeschichte, 42-43/2013 (https://www.bpb.de/apuz/170166/aufarbeitung-der-sed-diktatur-heute-so-wie-gestern? $p=a l l$, Zugriff am 01.11.2019).

Combe, Sonia (2019), „Usages et mésusages de la mémoire en Allemagne", in Le Monde, 28.02.2019, 29.

Combe, Sonia / Dufrêne, Thierry / Robin, Régine (2009), Berlin: L'effacement de traces, 1989-2009, Paris, BDIC/Fage Éditions.

Dahn, Daniela (2019), Der Schnee von gestern ist die Sintflut von heute. Die Einheit - eine Abrechnung, Hamburg, Rowohlt Taschenbuch Verlag.

Dell, Matthias (2019), „Ohnmachtspolitik. Ein Leipziger Lehrstück“, in Cargo. Film, Medien, Kultur, 30/10/2019 (https://www.cargo-film.de/festival/dok-leipzig/dok-leipzig-2019/dok-leipzig-2019/, Zugriff am 04.11.2019).

Dresen, Andreas / Stieler, Laila (2018), „Es gibt keine Absolution, Punkt", Interview mit Katja Nicodemus und Hanno Rauterberg, in Die Zeit, 03.10.2018 (https://www.zeit.de/2018/41/andreas-dresen-laila-stieler-gundermann-interview /komplett ansicht, Zugriff am 03.11.2019).

Engler, Wolfgang / Hensel, Jana (2018), Wer wir sind. Die Erfahrung, ostdeutsch zu sein, Berlin, Aufbau Verlag.

Fulbrook, Mary (2008), Ein ganz normales Leben. Alltag und Gesellschaft in der DDR, Darmstadt, WGB.

Frank, N. (2018), „Spirou in Berlin“, 21.09.2018 (https://www.arte.tv/ de/videos/085002-000-A/comic-spirou-in-ost-berlin/, Zugriff am 01.11.2019).
Goudin, Élisa (2017), „Destruction du Palais de la République et reconstruction du château des Hohenzollern: quelle place pour les considérations d'éthique publique dans la gestion du patrimoine berlinois?", in Éthique publique, Revue internationale d'éthique sociale et gouvernementale, (2) 19, (https://journals.openedition. org/ethiquepublique/2966, Zugriff am 30.10.2019).

Kollmorgen, Raj (2017), „Ostdeutsche in den Eliten. Problemdimensionen und Zukunftsperspektiven", in Ostdeutsche Eliten. Träume, Wirklichkeiten und Perspektiven, hrsg. v. Deutsche Gesellschaft e.V., Berlin, 54-65 (https://www.deutsche-gesellschaft-ev.de/ images/veranstaltungen/konferenzen-tagungen/2017-pb-ostdeutsche-eliten/Deutsche_Gesellschaft_eV_Broschuere_Ostdeutsche_Eliten.pdf, Zugriff am 03.11.2019).

Kott, Sandrine / Droit, Emmanuel (Hrsg.) (2006), Die ostdeutsche Gesellschaft. Eine transnationale Perspektive, Berlin, Christoph Links Verlag.

Lindenberger, Thomas (Hrsg.) (1999), Herrschaft und Eigen-Sinn in der Diktatur. Studien zur Gesellschaftsgeschichte der DDR, Böhlau, Köln/Weimar/Wien.

Mau, Steffen (2019): Lütten Klein. Leben in der ostdeutschen Transformationsgesellschaft, Berlin, Suhrkamp.

Mesnard, Philippe (2018), „Deux façons de raconter l'histoire“, in Mémoires en jeu, 7, 10-12. (https://www.memoires-en-jeu.com/actu/ deux-facons-de-raconter-Ihistoire/, Zugriff am 03.11.2019).

Moine, Caroline (2006), „Spur der Zeiten: images documentaires, images de fiction de la DEFA. Un autre regard sur la RDA?", in Fabre-Renault, Catherine / Goudin, Élisa / Hähnel-Mesnard, Carola (Hrsg.), La RDA au passé présent. Relectures critiques et réflexions pédagogiques, Paris, Presses Sorbonne Nouvelle, 171-189.

Robin, Régine (2001), Berlin, chantiers, Paris, Stock.

Sabrow, Martin / Eckert Rainer et al. (2007), Wohin treibt die DDR-Erinnerung? Dokumentation einer Debatte, Göttingen, Vandenhoeck \& Ruprecht.

Steinle, Matthias (2018), „Interroger les images de la RDA. Entretien avec le documentariste Thomas Heise", in Camarade / Guilhamon u.a., a.a.O., 303-318. 